\title{
Morphological parameters of middle cerebral arteries associated with aneurysm formation
}

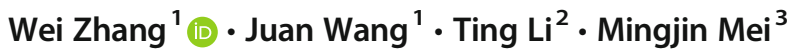 \\ Received: 17 May 2020 / Accepted: 13 August 2020 / Published online: 19 August 2020 \\ (C) The Author(s) 2020
}

\begin{abstract}
Purpose The objective of this work was to investigate the correlation between morphological parameters of the MCA and the formation of aneurysms.

Methods MCA aneurysms were diagnosed in 122 cases using CT angiography (including 30 cases of M1 proximal aneurysms, 70 cases of M1 bifurcation aneurysms, and 22 cases of distal aneurysms). Images from these cases were retrospectively compared with images from 50 healthy controls. Morphological parameters including the angle of the MCA with the ICA $(\alpha)$ and the ACA $(\beta)$ were evaluated in the three aneurysm groups and the control group; parent-daughter angles $\left(\gamma_{1}, \gamma_{2}\right)$, bifurcation angles $\left(\gamma_{3}\right)$, bifurcation diameters, angle ratios, and branch diameter ratios were also compared between the bifurcation aneurysm group and the control group. The blood vessel parameters between the aneurysm groups and controls were analyzed statistically.

Results There was no statistically significant difference in $\alpha$ between the three groups of aneurysms and the control group $(P=$ 0.381). In comparing $\beta$ between the three groups of aneurysms and the control group, statistically significant differences were only observed between the MCA distal aneurysm group and the control group $(P=0.010)$. Compared with the control group, MCA bifurcation aneurysms were associated with larger $\gamma_{3}$ and smaller $\gamma_{1}$ and $\gamma_{2}(P<0.001)$. This resulted in significantly larger angle ratios in the MCA bifurcation aneurysm group $(P<0.001)$. For the diameter measurements, the bifurcation diameter of the MCA bifurcation aneurysms was significantly smaller $(P=0.001)$.

Conclusion The formation of MCA aneurysms is related to morphological parameters.
\end{abstract}

Keywords Middle cerebral artery $\cdot$ Morphological parameters $\cdot$ Intracranial aneurysm $\cdot \mathrm{CT}$ angiography

\section{Introduction}

Intracranial aneurysms are characterized by a local abnormal expansion of an arterial wall. The incidence of intracranial aneurysms is relatively high, and approximately $50 \%$ of patients with subarachnoid hemorrhages caused by aneurysm rupture are left

Wei Zhang

1761955064@qq.com

1 Department of Radiology, Brain Hospital of Hunan Province, No. 427, Section 3, Furong Middle Road, Yuhua District, Changsha City, Hunan Province, China

2 Department of Radiology, The Third Xiangya Hospital of Central South University, No. 138 Tongzipo Road, Yuelu District, Changsha, Hunan, China

3 GE Healthcare Medical Affair, 10/F GE Tower, No. 87 Hua Cheng Avenue, Pearl River New City, Tianhe District,

Guangzhou, Guangdong, China with severe disability; furthermore, there is a $23 \sim 35 \%$ mortality rate when aneurysms are left untreated $[1,2]$. There are many reports on the mechanisms of intracranial aneurysm (IAs) formation, which are currently considered to be related to heredity, hemodynamics, hypertension, inflammatory response, and lipid metabolism disorders. There is evidence that genetic factors, such as genetic polymorphisms, play an important role in aneurysm formation. Wu et al. found that the LOXL2c.C133T mutation was a pathogenic gene by conducting complete exon sequencing of representative families with a history of IAs [3]. Theodotou et al. retrospectively analyzed the literature on cerebral aneurysm formation and genetic correlations from December 2008 to June 2015. Their work found that gene 9p21/CDKN2, mainly involved in vascular wall remodeling, had the strongest correlation with IAs. Other genes potentially related to aneurysm formation include EDNRA and SOX17 [4].

MCA aneurysms account for $20 \%$ of intracranial aneurysms, with a rupture rate of approximately 50\% [5]. MCA aneurysms are usually found after rupturing. They often result 
Fig. 1 Flowchart for inclusion of patients with MCA aneurysms

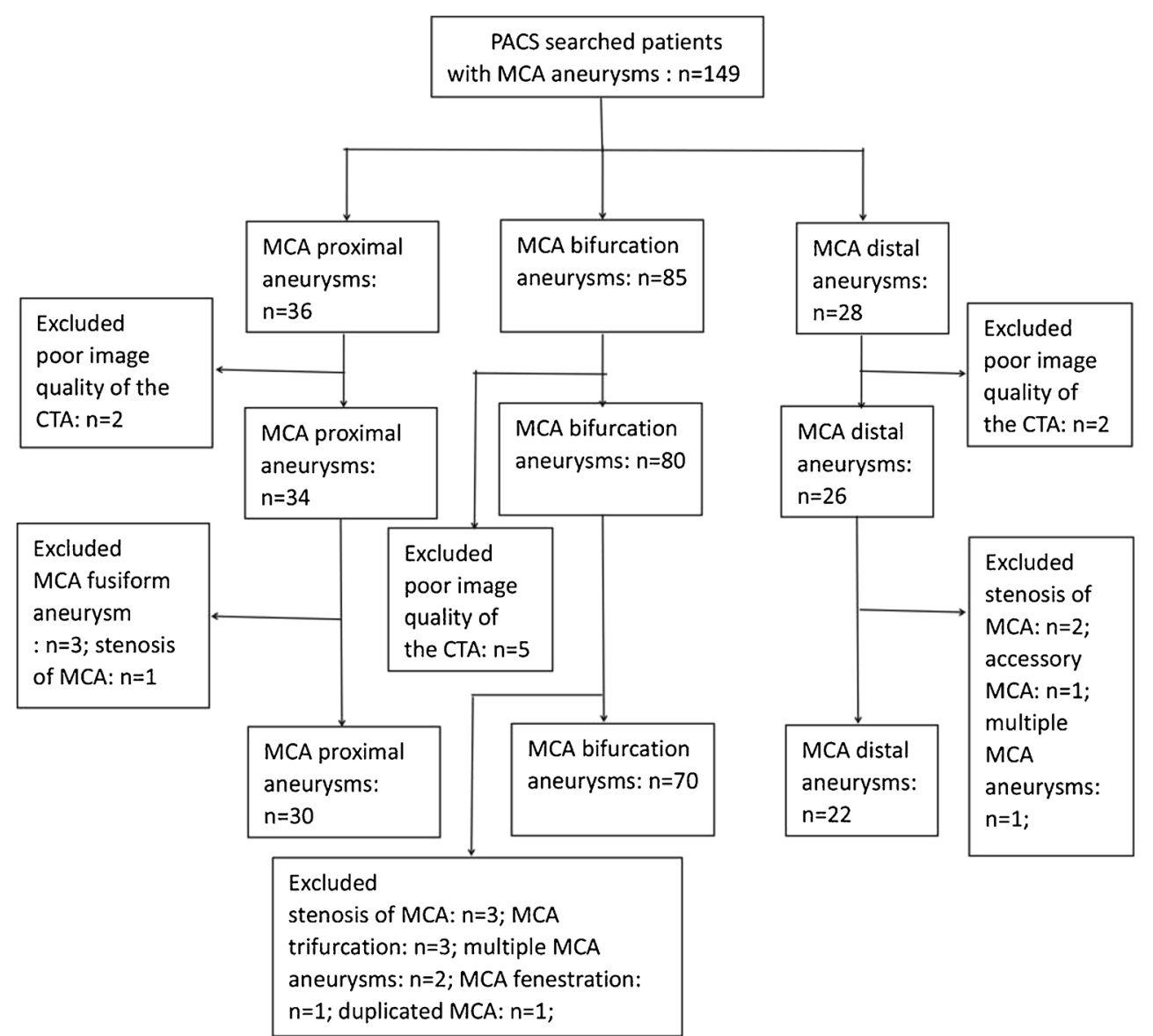

in intracranial hemorrhage and have higher mortality and disability rates compared with other IAs [6]. Therefore, it is of great importance to effectively screen high-risk groups regularly for early diagnosis and treatment of MCA aneurysms.

MCA aneurysms can be divided into three types according to their location: (1) proximal aneurysms located in the MCA prior to the bifurcation of the M1 segment (2-7\% of MCA aneurysms), (2) bifurcation aneurysms located in the first bifurcation of the MCA (80-85\% of MCA aneurysms), and (3) distal aneurysms located in the M2 segment and beyond (2 6\% of MCA aneurysms) [7-10].
Fig. 2 Angles and angle ratios were measured and defined as follows: ICA-MCA angle $(\alpha)$, ACA-MCA angle $(\beta), \gamma_{1}$ between the larger daughter vessel and parent vessel, $\gamma_{2}$ between the smaller vessel and parent vessel, $\gamma_{3}$ between the larger daughter vessel and the smaller vessel.

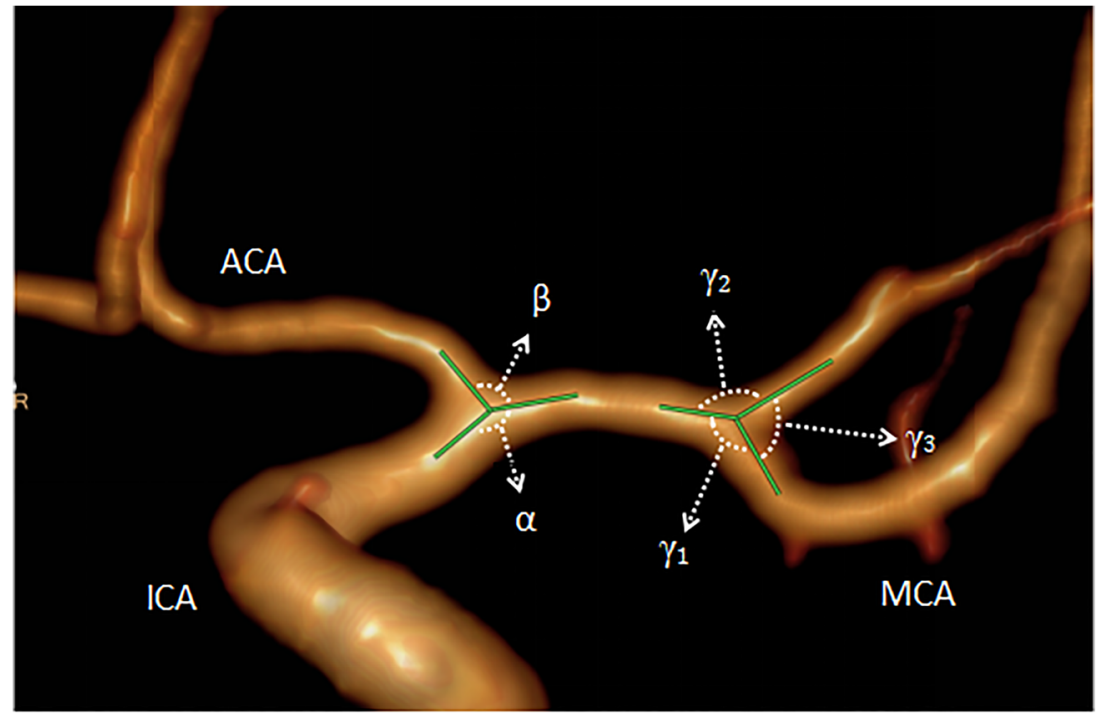


Fig. 3 Diameter and diameter ratios were measured and defined as follows: the diameter of the M1 segment bifurcation; the branch diameter ratio was calculated by the ratio of the larger daughter diameter to the smaller daughter diameter

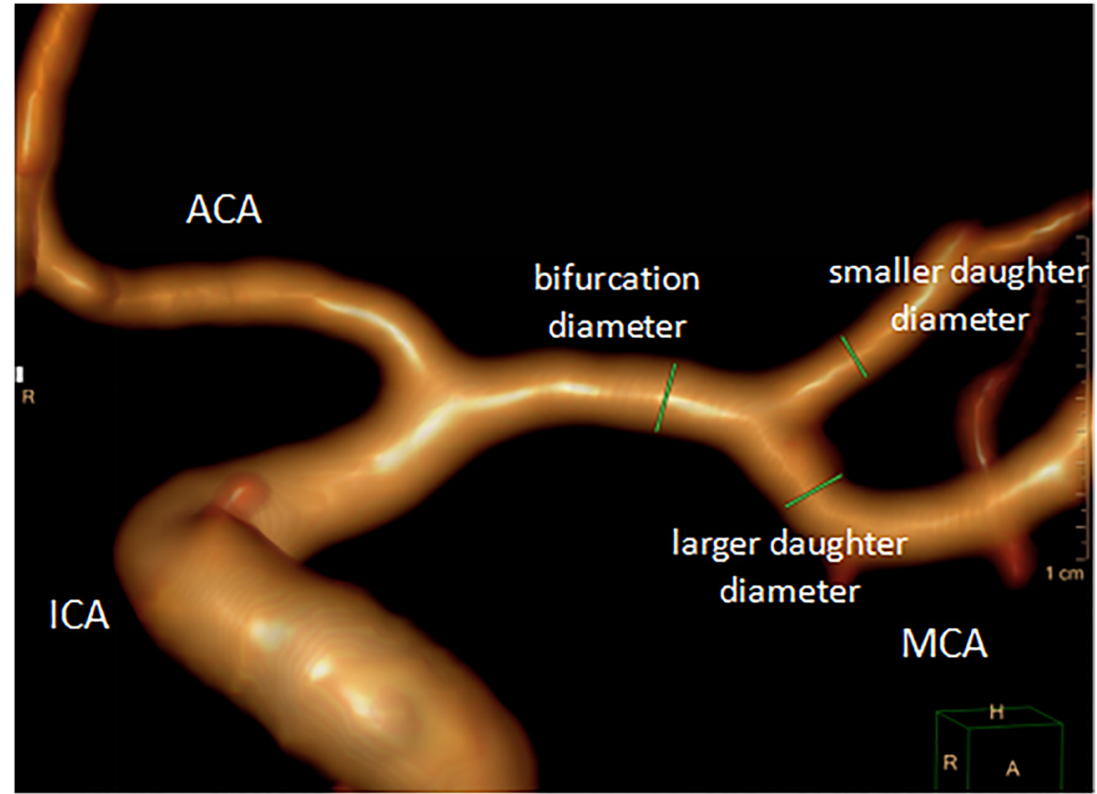

Due to the high incidence of MCA bifurcation aneurysms, many studies have focused on the formation of aneurysms as a result of morphological factors. These studies have confirmed that the formation of these aneurysms is related to the diameter and angle of the MCA bifurcation $[11,12]$, but did not study whether aneurysm formation was related to the morphological angle of the MCA with the internal carotid artery (ICA) and anterior cerebral artery (ACA). In addition, the association between the formation of proximal and distal aneurysms of the MCA and the angle between the MCA and ICA and ACA has not been previously reported. The bifurcation has special hemodynamic characteristics associated with an increased risk of aneurysm formation [13].

The purpose of this work was to study whether the angle of the MCA with the ICA and ACA contributes to the formation of MCA aneurysms. In our study, the correlation between
MCA geometry and aneurysm formation was investigated to provide references for aneurysm screening and risk assessment in high-risk patients.

\section{Methods}

\section{Patient selection}

This study was approved by the local medical ethics committee. Due to the retrospective nature of this study, informed consent was waived. A total of 122 patients with MCA aneurysms and 50 patients without aneurysms were enrolled from January 2014 to September 2019. The MCA aneurysm group was divided into three subgroups: (1) M1 segment proximal aneurysm $(n=30),(2)$ M1 segment bifurcation aneurysm $(n=$ $70)$, and (3) distal aneurysm $(n=22)$. Inclusion criteria in the
Table 1 Summary of all patient's clinical data

\begin{tabular}{lllllll}
\hline & $\begin{array}{l}\text { Control } \\
(n=50)\end{array}$ & $\begin{array}{l}\text { MCA proximal } \\
\text { aneurysms }(n= \\
30)\end{array}$ & $\begin{array}{l}\text { MCA bifurcation } \\
\text { aneurysms }(n= \\
70)\end{array}$ & $\begin{array}{l}\text { MCA distal } \\
\text { aneurysms }(n= \\
22)\end{array}$ & $\begin{array}{l}F / ?^{2} \\
\text { value }\end{array}$ \\
\hline Age (years) & $\begin{array}{c}56.36 \pm \\
13.51\end{array}$ & $54.53 \pm 12.05$ & $60.42 \pm 10.23$ & $57.32 \pm 16.05$ & 1.198 & 0.119 \\
Gender M/F & $33: 17$ & $16: 14$ & $24: 46$ & $6: 16$ & 5.401 & 0.001 \\
$\begin{array}{l}\text { Diabetes(N/Y) } \\
\text { Hypertension(N/Y) }\end{array}$ & $33: 17$ & $16: 14$ & $36: 34$ & $11: 11$ & 3.026 & 0.388 \\
$\begin{array}{l}\text { Hyperlipidemia } \\
\text { (N/Y) }\end{array}$ & $28: 22$ & $14: 16$ & $19: 51$ & $7: 15$ & 2.324 & 0.508 \\
\hline
\end{tabular}

$M$ male, $F$ female, $N$ without, $Y$ with 


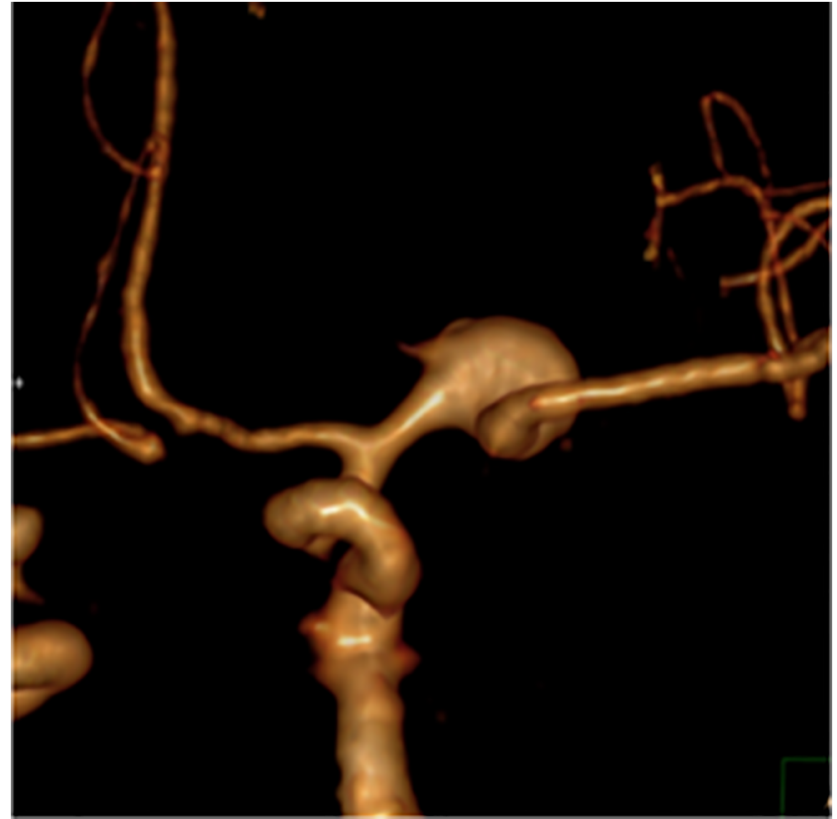

Fig. 4 An example of an anterior view of a 3D rendered MCA proximal aneurysm is shown

control group were absence of intracranial arteriovenous malformations, Moyamoya disease, subarachnoid hemorrhage, vasospasm, intracranial hemorrhage, or Circle of Willis loop aneurysms. The exclusion criteria of the aneurysm group were (1) fusiform aneurysm of the MCA or arteriovenous malformations, (2) stenosis of MCA vessels, (3) multiple MCA aneurysms, MCA trifurcation, early bifurcation, duplicated MCA, accessory MCA, or fenestration, and (4) poor image quality of the CTA examination. The flowchart of the patients with MCA aneurysms included in the analysis is shown in Fig. 1.

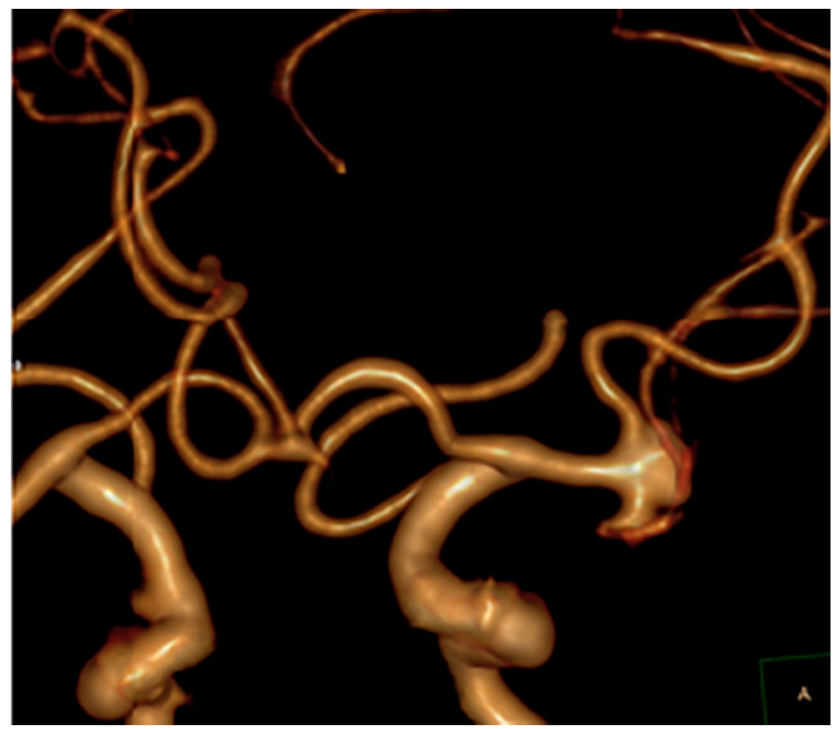

Fig. 5 An example of an anterior view of a 3D rendered MCA bifurcation aneurysm is shown

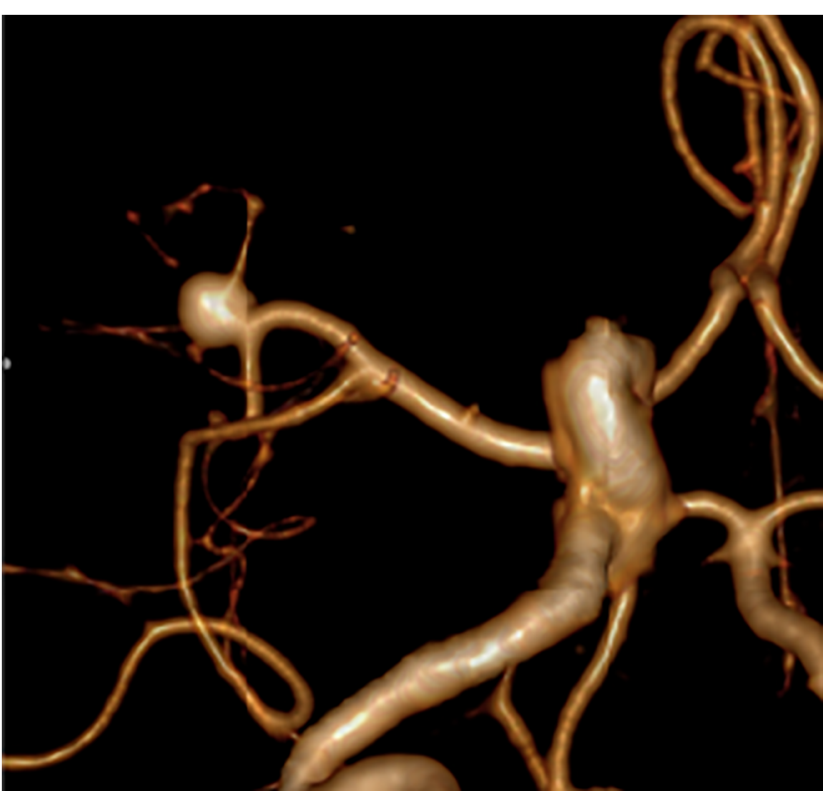

Fig. 6 An example of a posterior view of a 3D rendered MCA distal aneurysm is shown

\section{Data acquisition}

All subjects were scanned using three-dimensional CTA with 128-slice CT (Philips Ingenuity Core, scanning parameters: $120 \mathrm{kVp}, 240 \mathrm{mAs}$, contrast material: iopromide $370 \mathrm{mg} / \mathrm{ml}$, injection speed $5.0 \mathrm{ml} / \mathrm{s}$, dose $50 \mathrm{ml}$ ) or 16-row CT (GE Bright Speed, scanning parameters: $120 \mathrm{kVp}, 300 \mathrm{mAs}$, contrast material: iopromide $370 \mathrm{mg} / \mathrm{ml}$, injection speed $4.0 \mathrm{ml} / \mathrm{s}$, dose 60 $\mathrm{ml})$. Images were transmitted to Philips Extended Brilliance Workspace for vascular reconstruction, bone removal and silhouette, and three-dimensional volume rendering (3D VR) and maximum-intensity projection (MIP). We measured by Philips Advanced Vessel Analysis Software. The vessel diameter was measured by using VR image, and the window setting for VR image was integrated based on the full width at half maximum on maximum intensity projection. Each dataset was manually measured by two primary physicians, who in turn performed each measurement twice. The average of these two measurements was used for analysis.

\section{Definition of morphological parameters}

See Figs. 2 and 3 for definitions of morphologic measurements. All angles were measured on a three-dimensional longitudinal section of the center of the two vessels of interest. These measured angles included the following: between the MCA and ICA $-\alpha$; between the MCA and ACA $-\beta$; bifurcation angle between the larger daughter vessel and parent vessel $-\gamma_{1}$; between the smaller vessel and parent vessel$\gamma_{2}$; and between the larger daughter vessel and smaller vessel- $\gamma_{3}$. Angle ratio was calculated as the ratio of larger parent-daughter angle to smaller parent-daughter angle. 
Table 2 Comparison of vascular morphological parameters between three groups of MCA aneurysms and the control group

\begin{tabular}{|c|c|c|c|c|c|c|}
\hline & \multirow{2}{*}{$\begin{array}{l}\text { Control } \\
(n=50)\end{array}$} & \multicolumn{3}{|l|}{ Aneurysms $(n=122)$} & \multirow{2}{*}{$\begin{array}{l}H \\
\text { value }\end{array}$} & \multirow{2}{*}{$\begin{array}{l}P \\
\text { value }\end{array}$} \\
\hline & & $\begin{array}{l}\text { MCA proximal } \\
\text { aneurysms }(n=30)\end{array}$ & $\begin{array}{l}\text { MCA bifurcation } \\
\text { aneurysms }(n=70)\end{array}$ & $\begin{array}{l}\text { MCA distal } \\
\text { aneurysms }(n= \\
22)\end{array}$ & & \\
\hline$\alpha\left({ }^{\circ}\right)$ & $\begin{array}{l}134.50 \\
\quad(17.2- \\
5)\end{array}$ & $139.50(23.50)$ & $140.00(21.25)$ & $143.00(16.00)$ & 3.072 & 0.381 \\
\hline$\beta\left({ }^{\circ}\right)$ & $\begin{array}{l}120.00 \\
(19.2- \\
5)\end{array}$ & $111.50(29.25)$ & $118.00(28.25)$ & $109.00(19.00)$ & 8.047 & 0.045 \\
\hline
\end{tabular}

$\alpha$ and $\beta$ using Kruskal-Wallis $H$ test. Shown are median (interquartile range)
Diameter measurements were taken in the M1 segment bifurcation approximately 2 vessel diameters from the bifurcation [14]. The branch diameter ratio was calculated as the ratio of the larger daughter diameter to the smaller daughter diameter.

\section{Statistical analysis}

In the control group and the bifurcation aneurysm group, $\gamma_{1}$, $\gamma_{2}, \gamma_{3}$, the bifurcation diameter branch, and the diameter ratio were tested independently using a $t$ test for normally distributed data; angle ratios were tested using a Wilcoxon rank-sum test for non-normally distributed data. The Kruskal-Wallis $\mathrm{H}$ test was used for $\alpha$ and $\beta$ between the control group and the three groups of aneurysm, respectively. Receiver operating

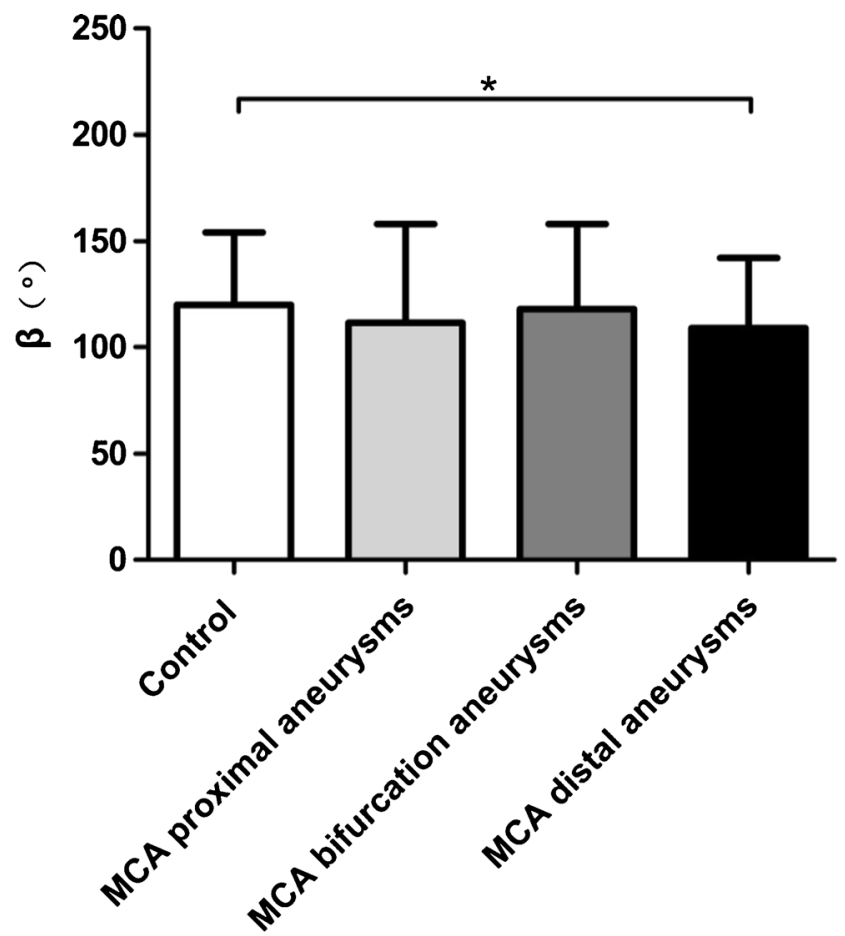

Fig. 7 The MCA-ACA angle ( $\beta$ ) between the three aneurysm groups and the control group is plotted as bar graphs with standard deviation error bars. $* p<0.05, * * p<0.001$ characteristic (ROC) curves of morphological parameters with statistical significance were obtained to determine cut-off values and then to evaluate the sensitivity and specificity of diagnosis. Age, sex, hypertension, diabetes, and hyperlipidemia were analyzed for differences between the control group and three aneurysm groups by using the $\chi^{2}$ test. Across all tests, $P<0.050$ indicated a statistically significant difference. SPSS release 20.0 software (IBM Corp, Armonk, New York) was used for the statistical analysis.

\section{Results}

The study included 33 males and 17 females in the control group. The mean ( \pm standard deviation) age of the patients in the control group was $56.36( \pm 13.51)$ years, ranging from 22 to 81 years. The three aneurysm groups included 46 males and 76 females. The mean age of the patients in the three aneurysm groups was $58.42( \pm 12.06)$ years, ranging from 25 to 88 years. There was no significant difference in age between the three MCA aneurysm groups and the control group. Compared with the control group, the number of females in aneurysm groups was significantly higher than the number of men $(P=0.001)$. And statistical analysis of the clinical factors of hypertension, diabetes, and hyperlipidemia is presented in Table 1.

3D renderings of each type of aneurysm are shown in Figs. 4, 5, and 6 and depict excellent delineation of the vessel and aneurysm wall in all subtypes.

At the MCA-ACA junction (Table 2, Fig. 7), there was no statistically significant difference in $\alpha$ between the three groups of aneurysms and the control group $(P=0.381)$. In comparing $\beta$ between the three groups of aneurysms and the control group, statistically significant differences were only observed between the MCA distal aneurysm group and the control group (median $109.00^{\circ}\left(19.00^{\circ}\right)$ vs. $120.00^{\circ}$ $\left.\left(19.25^{\circ}\right), P=0.010\right)$.

Statistical analysis results comparing the MCA bifurcation aneurysm group and the control group are shown in Table 3 
Table 3 Comparison of vascular morphological parameters between MCA bifurcation aneurysms and the control group

\begin{tabular}{lllll}
\hline & Control $(n=50)$ & MCA bifurcation aneurysms $(n=70)$ & $t / Z$ value & $P$ value \\
\hline$\gamma_{1}\left({ }^{\circ}\right)^{\mathrm{a}}$ & $128.78 \pm 16.54$ & $109.59 \pm 30.68$ & 4.336 & $<0.001$ \\
$\gamma_{2}\left({ }^{\circ}\right)^{\mathrm{a}}$ & $117.33 \pm 22.16$ & $87.21 \pm 27.46$ & 6.221 & $<0.001$ \\
$\gamma_{3}\left({ }^{\circ}\right)^{\mathrm{a}}$ & $95.45 \pm 19.55$ & $143.74 \pm 36.57$ & -8.136 & $<0.001$ \\
${\text { Bifurcation diameter }(\mathrm{mm})^{\mathrm{a}}}^{\mathrm{a}}$ & $2.53 \pm 0.53$ & $2.18 \pm 0.56$ & 3.374 & 0.001 \\
Angle ratio $^{\mathrm{b}}$ & $1.13(0.27)$ & $1.46(0.77)$ & -4.000 & $<0.001$ \\
Branch diameter ratio $^{\mathrm{a}}$ & $1.49 \pm 0.45$ & $1.49 \pm 0.38$ & 0.101 & 0.920 \\
\hline
\end{tabular}

${ }^{\mathrm{a}} t$ test, shown are mean and standard deviation

${ }^{\mathrm{b}}$ Wilcoxon rank-sum test, shown are the median (interquartile range) and Fig. 8. Compared with the control group, MCA bifurcation aneurysms were associated with larger $\gamma_{3}$ (mean $143.74^{\circ}$ $\pm 36.57^{\circ}$ vs. $95.45^{\circ} \pm 19.55^{\circ}, P<0.001$ ) and smaller $\gamma_{1}$ (mean $109.59^{\circ} \pm 30.68^{\circ}$ vs. $\left.128.78^{\circ} \pm 16.54^{\circ}, P<0.001\right)$ and $\gamma_{2}$ (mean $87.21^{\circ} \pm 27.46^{\circ}$ vs. $117.33^{\circ} \pm 22.16^{\circ}, P<0.001$ ). This resulted in significantly larger angle ratios in the MCA bifurcation aneurysm group (mean $1.46 \pm 0.77$ vs. $1.13 \pm 0.27$, $P<0.001)$. For the diameter measurements, the bifurcation diameter of the MCA bifurcation aneurysms was significantly smaller (mean $2.18 \pm 0.56$ vs. $2.53 \pm 0.53, P=0.001$ ), and there was no statistical difference in the branch diameter ratio $(P=0.920)$.

The ROC curve analysis (Table 4, Fig. 9) results showed that the AUC of $\gamma_{1}, \gamma_{2}, \gamma_{3}$, the bifurcation diameter in the MCA bifurcation aneurysm group, and $\beta$ in the MCA distal aneurysm group were all greater than 0.7 . Meanwhile, the cutoff value of $\gamma_{3}=104.50^{\circ}$ had the highest diagnostic accuracy (AUC $=0.881$ ), with a sensitivity of $78.26 \%$ and a specificity of $88.57 \%$.

\section{Discussion}

As early as 1930 , scholars proposed that intracranial arterial morphology and hemodynamic changes were extremely important factors in the formation and development of aneurysms. Aneurysm geometry and hemodynamics interact with each other through blood flow (i.e., wall shear stress (WSS) and blood pressure) to determine the future geometry of an aneurysm (enlargement and morphological change) [15]. In this study, we aimed to study the correlation between the formation of MCA aneurysms and morphological parameters.

\section{Morphological characteristics of MCA bifurcation aneurysms and the contro}

Several previously published studies support the findings presented here. Hademeno et al. showed that the direction of blood flow in the bifurcation of the vessel deviates, and the impact force and shear force on the bifurcation are significantly increased [16]. Their study confirmed that pressure could be 2-3 times that of adjacent arteries with aneurysms. Can et al. studied the bifurcation diameter and bifurcation angle between 73 MCA bifurcation aneurysm cases and 37 controls. In agreement with our results, they showed that the bifurcation diameter in aneurysm patients was smaller than in controls, while the bifurcation angle was larger [17]. Jianping et al. found that the most relevant parameter for the formation of MCA bifurcated aneurysms was the bifurcation angle, and its cut-off value was $124.8^{\circ}$ [18]. In our study, $\gamma_{3}$ also had the highest diagnostic accuracy (AUC $=0.881$ ), but the cut-off value was $104.5^{\circ}$, a discrepancy that may be due to research protocol differences. Baharoglu et al. found that when the MCA branch inclination angle increases, the blood vessels turn sharply, thus deviating the inflowing blood and potentially leading to the formation of aneurysms [19]. Similarly, $\gamma_{1}$ and $\gamma_{2}$ were also associated with the formation of MCA aneurysms in our study. This may be due to the expansion of the bifurcation angle and the MCA branch inclination angle causing the WSS and wall shear stress gradient (WSSG) of vessels to increase.

Previous studies have explored that MCA bifurcation aneurysm formation is related to the angle of bifurcation, but whether it is related to $\alpha$ and $\beta$ has rarely been reported. At present, only Duan et al. showed that $\alpha$ in the MCA bifurcation aneurysm group was smaller than the control group (mean $130^{\circ} \pm 15^{\circ}$ vs. $133^{\circ} \pm 12^{\circ}, P=0.01$ ) [20]. But in our study, there was no statistical difference in $\alpha$ between groups. At the same time, we found that $\beta$ was not associated with the formation of bifurcation aneurysms. We speculate that the large distance between $\alpha, \beta$, and the MCA bifurcation may have little effect on the blood flow pressure of the bifurcation. Thus, there is no obvious correlation with the formation of a bifurcation aneurysm.

Fig. 8 Results of $\gamma_{1}(\mathbf{a}), \gamma_{2}$ (b), $\gamma_{3}$ (c), the bifurcation diameter (d), and the angle ratio (e) between the MCA bifurcation aneurysm group and the control group are plotted as bar graphs with standard deviation error bars. $* p<0.05, * * p<0.001$ 
a

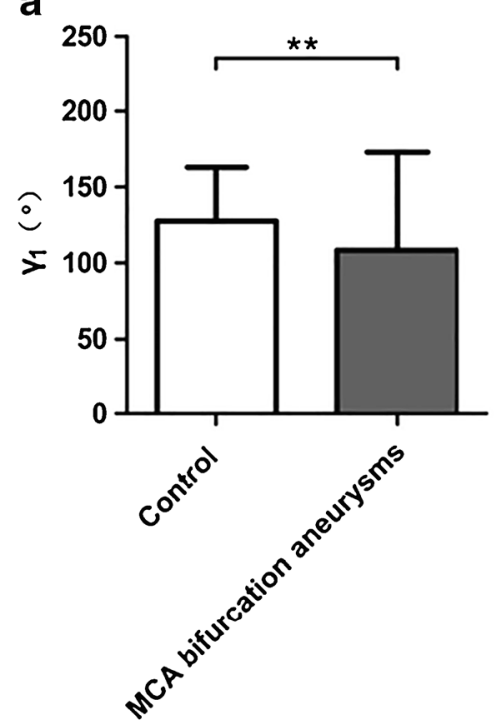

c

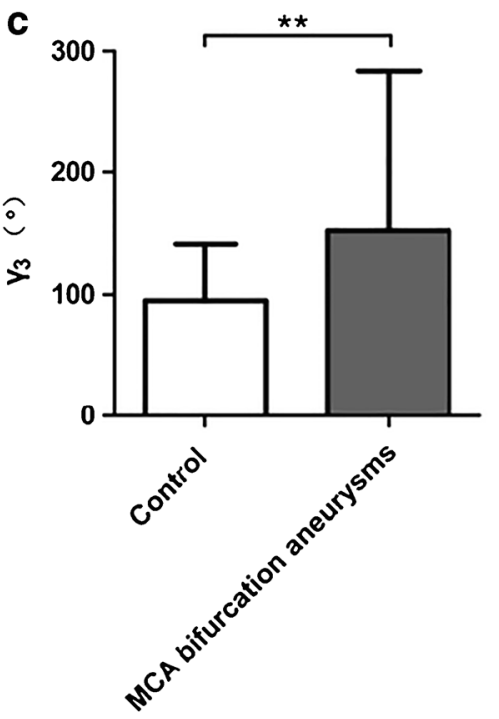

e

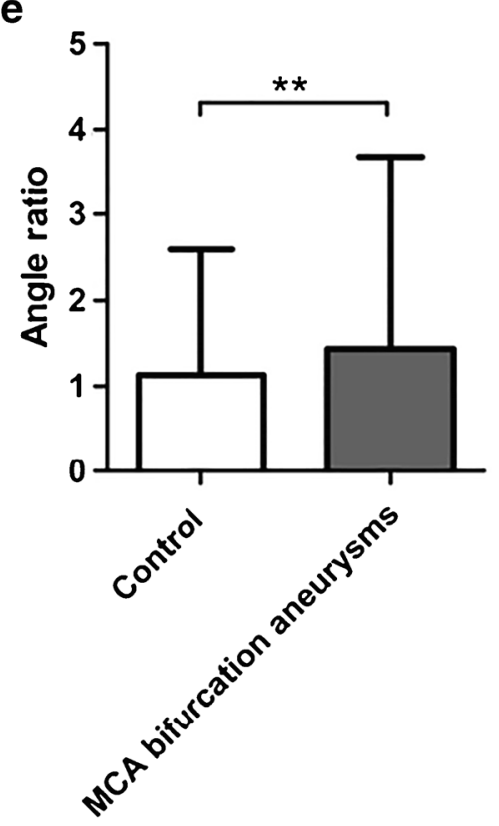

b

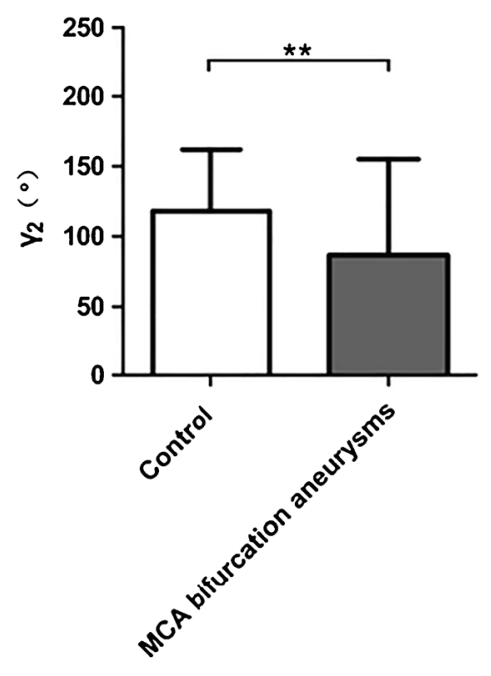

d

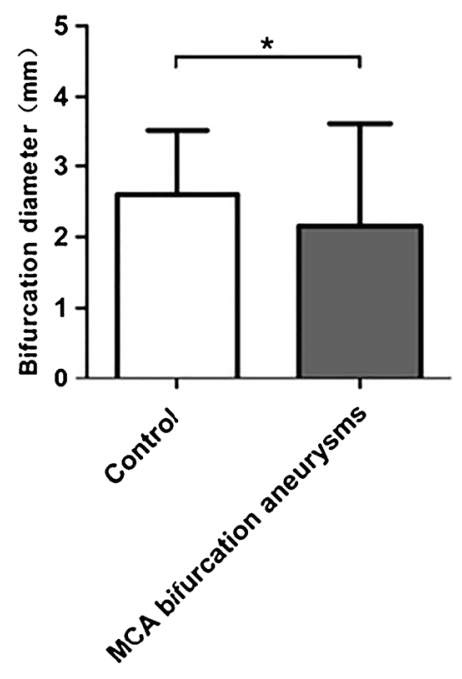


Table 4 Comparison of diagnostic efficacy of various vascular morphological parameters

\begin{tabular}{llllll}
\hline & Cutoff score & Sensitivity $(\%)$ & Specificity $(\%)$ & AUC & $95 \%$ CI \\
\hline $\begin{array}{l}\text { MCA distal aneurysms } \\
\beta\left({ }^{\circ}\right)\end{array}$ & 113.50 & 72.00 & 70.00 & 0.713 & $0.571 \sim 0.854$ \\
MCA bifurcation aneurysms & & & & & \\
$\gamma_{1}\left({ }^{\circ}\right)$ & 103.50 & 93.48 & 47.14 & 0.717 & $0.624 \sim 0.809$ \\
$\gamma_{2}\left({ }^{\circ}\right)$ & 98.00 & 86.96 & 74.29 & 0.812 & $0.731 \sim 0.893$ \\
$\gamma_{3}\left({ }^{\circ}\right)$ & 104.50 & 78.26 & 88.57 & 0.881 & $0.818 \sim 0.945$ \\
Bifurcation diameter(mm) & 2.45 & 60.87 & 67.14 & 0.720 & $0.623 \sim 0.812$ \\
Angle ratio & 1.63 & 93.48 & 41.43 & 0.686 & $0.587 \sim 0.784$ \\
\hline
\end{tabular}

The morphological parameters of the MCA bifurcation also include the vascular diameter. Smaller diameter vessels may be subjected to relatively strong hemodynamic stress, or the walls of smaller vessels may be too thin to withstand the said hemodynamic stress; both of these factors lead to the formation of aneurysms. Alexandra et al. showed that smooth blood vessels in patient models gradually thinned, which also led to the acceleration of blood flow at the MCA bifurcation, increased WSS and WSSG at the bifurcation vertex, and resulted in the formation of bifurcation aneurysms [21]. Bor et al. measured the geometric parameters of 104 cases of MCA bifurcation vessels and found that the incidence of aneurysms was higher in branch dysplasia (diameter $<1 \mathrm{~mm}$ ) [22]. This agrees well with findings which point to the diameter cut-off value of $2.45 \mathrm{~mm}$.

\section{Morphological features between MCA proximal and distal aneurysms and the control}

A majority ( $80 \%$ to $85 \%$ ) of MCA aneurysms occur at the bifurcation [23]. MCA proximal and distal aneurysms are relatively rare, and most previous studies of proximal and distal aneurysms focus on the selection of treatment options for aneurysm rupture. Few studies exist on the relationship between MCA geometry and the formation of proximal and distal aneurysms [24-27]. To the best of our knowledge, the relation of
Fig. 9 Receiver operator curves are plotted as a comparison of diagnostic efficacy for the measured vascular morphological parameters. a $\beta$. b $\gamma_{1}, \gamma_{2}, \gamma_{3}$, the bifurcation diameter, and the angle ratio
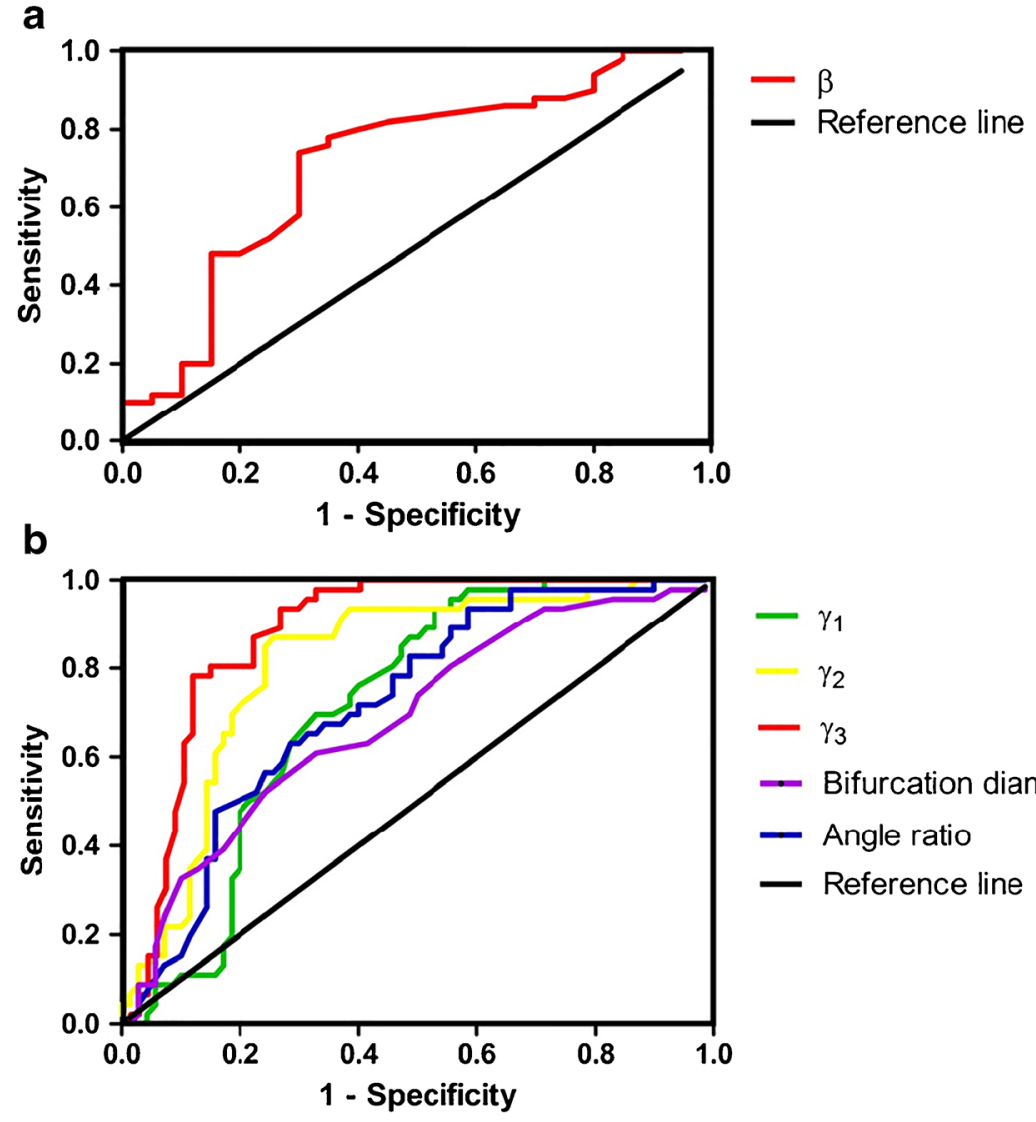
both the $\alpha$ and $\beta$ to the formation of proximal and distal MCA aneurysms has not yet been reported. We compared and analyzed $\alpha$ and $\beta$ between an MCA proximal aneurysm group, distal aneurysm group, and control group and found that there was no significant correlation between proximal aneurysm formation and $\alpha$ and $\beta$, while $\beta$ in the distal aneurysm group was smaller than in controls. This difference was statistically significant (median $109.00^{\circ}\left(19.00^{\circ}\right)$ vs. $120.00^{\circ}\left(19.25^{\circ}\right), P=$ 0.010 ). When the cut-off value of $\beta$ was $113.5^{\circ}$, the sensitivity and specificity of diagnosis were highest. We speculate that this correlation may be related to hemodynamics, but due to the small sample size, further studies are needed. However, there was no significant correlation between $\alpha$ and MCA distal aneurysm formation.

\section{Limitations}

One limitation of this study is its retrospective nature. Although we have shown that the formation of MCA aneurysms is related to geometric shape, this does not directly suggest causality. Whether a difference in vascular morphology precipitates hemodynamic changes which then leads to the formation of aneurysms or whether peripheral vascular morphology changes during the formation of aneurysms is still unknown. Probing this question will require further studies.

\section{Conclusion}

Our study focused on the effects of morphological aspects of the formation of MCA aneurysms. $\gamma_{1}, \gamma_{2}, \gamma_{3}$, angle ratios, and bifurcation diameters were the relevant factors for the formation of MCA bifurcation aneurysms. MCA distal aneurysm formation was associated with $\beta$. The above parameters can be used to evaluate the susceptibility of aneurysms to rupture, which would facilitate the screening of high-risk groups of aneurysms.

Authors' contributions All authors contributed to the study conception and design. Material preparation, data collection, and analysis were performed by Juan Wang, Ting Li, and Mingjin Mei. The first draft of the manuscript was written by Wei Zhang and all authors commented on previous versions of the manuscript. All authors read and approved the final manuscript.

Funding information This study was funded by grants from Health and Family Planning Commission of Hunan Province, China ( B2016027).

Data availability Not applicable.

\section{Compliance with ethical standards}

Conflict of interest The authors declare that they have no conflicts of interest.
Ethical approval This retrospective study was approved by the ethics committee of the Brain Hospital of Hunan Province.

Informed consent This retrospective study was approved by the ethics committee of Hunan Brain Hospital to waive the informed consent of the patients.

Consent for publication All authors agree to be published

Code availability Not applicable.

Open Access This article is licensed under a Creative Commons Attribution 4.0 International License, which permits use, sharing, adaptation, distribution and reproduction in any medium or format, as long as you give appropriate credit to the original author(s) and the source, provide a link to the Creative Commons licence, and indicate if changes were made. The images or other third party material in this article are included in the article's Creative Commons licence, unless indicated otherwise in a credit line to the material. If material is not included in the article's Creative Commons licence and your intended use is not permitted by statutory regulation or exceeds the permitted use, you will need to obtain permission directly from the copyright holder. To view a copy of this licence, visit http://creativecommons.org/licenses/by/4.0/.

\section{References}

1. Li L, Sima X, Bai P et al (2012) Interactions of miR-34b/c and TP53 polymorphisms on the risk of intracranial aneurysm. Clin Dev Immunol 2012:567586

2. Hoi Y, Meng H, Woodward SH, Bendok BR, Hanel RA, Guterman LR, Hopkins LN (2004) Effects of arterial geometry on aneurysm growth: three-dimensional computational fluid dynamics study. J Neurosurg 101:676-681

3. Wu Y, Li Z, Shi Y, Chen L, Tan H, Wang Z, Yin C, Liu L, Hu J (2018) Exome sequencing identifies LOXL2 mutation as a cause of familial intracranial aneurysm. World Neurosurg 109:e812-e818

4. Theodotou CB, Snelling BM, Sur S, Haussen DC, Peterson EC, Elhammady MS (2017) Genetic associations of intracranial aneurysm formation and sub-arachnoid hemorrhage. Asian J Neurosurg $12: 374-381$

5. Millon D, Derelle AL, Omoumi P, Tisserand M, Schmitt E, Foscolo S, Anxionnat R, Bracard S (2012) Nontraumatic subarachnoid hemorrhage management: evaluation with reduced iodine volume at CT angiography. Radiology 264:203-209

6. Wong GKC, Boet R, Ng SCP, Chan M, Gin T, Zee B, Poon WS (2012) Ultra-early (within 24 hours) aneurysm treatment after subarachnoid hemorrhage. World Neurosurg 77:311-315

7. Ni W, Yang H, Xu B, Xu F, Jiang H, Lei Y, Su J, Gu Y, Mao Y (2019) Proximal middle cerebral artery aneurysms: microsurgical management and therapeutic results. World Neurosurg 122:e907-e916

8. Joo S-P, Kim T-S, Choi J-W, Lee JK, Kim YS, Moon KS, Kim JH, Kim SH (2007) Characteristics and management of ruptured distal middle cerebral artery aneurysms. Acta Neurochir (Wien) 149:661667

9. Brzegowy P, Polak J, Wnuk J, Łasocha B, Walocha J, Popiela TJ (2018) Middle cerebral artery anatomical variations and aneurysms: a retrospective study based on computed tomography angiography findings. Folia Morphol (Warsz) 77:434-440

10. Paulo M-S, Edgardo S, Fernando M et al (2010) Aneurysms of the middle cerebral artery proximal segment (M1) - anatomical and therapeutic considerations ' revision of a series. Analysis of a series of the pre bifurcation segment aneurysms. Asian J Neurosurg 5:57-63 
11. Ingebrigtsen T, Morgan MK, Faulder K, Ingebrigtsen L, Sparr T, Schirmer H (2004) Bifurcation geometry and the presence of cerebral artery aneurysms. J Neurosurg 101:108-113

12. Sadatomo T, Yuki K, Migita K, Imada Y, Kuwabara M, Kurisu K (2013) Differences between middle cerebral artery bifurcations with normal anatomy and those with aneurysms. Neurosurg Rev 36: 437-445

13. Alnaes MS, Isaksen J, Mardal K-A et al (2007) Computation of hemodynamics in the circle of Willis. Stroke 38:2500-2505

14. Baharoglu MI, Lauric A, Wu C, Hippelheuser J, Malek AM (2014) Deviation from optimal vascular caliber control at middle cerebral artery bifurcations harboring aneurysms. J Biomech 47:3318-3324

15. Meng H, Tutino VM, Xiang J, Siddiqui A (2014) High WSS or low WSS? Complex interactions of hemodynamics with intracranial aneurysm initiation, growth, and rupture: toward a unifying hypothesis. AJNR Am J Neuroradiol 35:1254-1262

16. Hademenos GJ, Massoud TF (1997) Biophysical mechanisms of stroke. Stroke 28:2067-2077

17. Can A, Ho AL, Dammers R, Dirven CMF, du R (2015) Morphological parameters associated with middle cerebral artery aneurysms. Neurosurgery 76:721-726 discussion 726-727

18. Song J, Zhu F, Qian Y, Ou C, Cai J, Zou X, Wu Z, Zhu W, Chen L, Mao Y (2017) Morphological and hemodynamic differences between aneurysmal middle cerebral artery bifurcation and contralateral nonaneurysmal Anatomy. Neurosurgery 81:779-786

19. Baharoglu MI, Lauric A, Safain MG, Hippelheuser J, Wu C, Malek AM (2014) Widening and high inclination of the middle cerebral artery bifurcation are associated with presence of aneurysms. Stroke 45:2649-2655

20. Duan Y, Lagman C, Ems R, Bambakidis NC (2019) Relationship between middle cerebral parent artery asymmetry and middle cerebral artery aneurysm rupture risk factors. J Neurosurg: $1-8$
21. Lauric A, Greim-Kuczewski K, Antonov A, Dardik G, Magida JK, Hippelheuser JE, Kono K, Malek AM (2019) Proximal parent vessel tapering is associated with aneurysm at the middle cerebral artery bifurcation. Neurosurgery 84:1082-1089

22. Bor ASE, Velthuis BK, Majoie CB, Rinkel GJE (2008) Configuration of intracranial arteries and development of aneurysms: a follow-up study. Neurology 70:700-705

23. Heros RC, Fritsch MJ (2001) Surgical management of middle cerebral artery aneurysms. Neurosurgery 48:780-785

24. Ding D, Bok AP (2017) Acute subdural hematoma from a ruptured aneurysm of the distal middle cerebral artery. J Neurosci Rural Pract 8:152-154

25. Sakata Y, Hadeishi H, Tanaka M (2017) Clinical characteristics and surgical approach to distal middle cerebral artery aneurysms. Surgery for Cerebral Stroke. 45:183-188

26. Chen F, Fang X (2019) Endovascular treatment of middle cerebral artery aneurysm with a (LVIS) device: comparison of LVIS stent and non-LVIS stent. Exp Ther Med 17:1656-1662

27. Primiani CT, Ren Z, Kan P, Hanel R, Pereira VM, Lui WM, Goyal N, Elijovich L, Arthur AS, Hasan DM, Ortega-Gutierrez S, Samaniego EA, Puri AS, Kuhn AL, Orlov K, Kislitsin D, Gorbatykh A, Waqas M, Levy EI, Siddiqui AH, Mokin M (2019) A2, M2, P2 aneurysms and beyond: results of treatment with pipeline embolization device in 65 patients. J Neurointerv Surg 11:903907

Publisher's note Springer Nature remains neutral with regard to jurisdictional claims in published maps and institutional affiliations. 